\title{
Entre « loi des Miao » et loi sur les Miao : le cas du trafic d'êtres humains dans le Guizhou au XVIII ${ }^{\mathrm{e}}$ siècle
}

Between «Miaoli » and Codified Laws on the Miao: The Trafficking in Human

Beings in 18th-Century Guizhou

清代苗疆控制中的苗例研究: 以整飭跨省人口交易為例

\section{Ning Laure Zhang}

\section{OpenEdition}

\section{Journals}

Édition électronique

URL : http://journals.openedition.org/extremeorient/605

DOI : 10.4000/extremeorient.605

ISBN : 978-2-84292-558-1

ISSN : 2108-7105

\section{Éditeur}

Presses universitaires de Vincennes

Édition imprimée

Date de publication : 21 novembre 2016

Pagination : 79-102

ISBN : 978-2-84292-556-7

ISSN : 0754-5010

\section{Référence électronique}

Ning Laure Zhang, «Entre « loi des Miao » et loi sur les Miao : le cas du trafic d'êtres humains dans le Guizhou au XVIII' siècle », Extrême-Orient Extrême-Occident [En ligne], 40 | 2016, mis en ligne le 21 novembre 2018, consulté le 30 avril 2019. URL : http://journals.openedition.org/extremeorient/605 ; DOI : 10.4000/extremeorient.605 


\title{
Entre « loi des Miao » et loi sur les Miao : le cas du trafic d'êtres humains dans le Guizhou au XVIII' siècle
}

\author{
Zhang Ning (Laure)
}

L'entreprise de conquête menée par la dynastie mandchoue dans le Sud de la Chine a pour effet la mise en place progressive d'une législation particulièrement complexe. En effet, elle devait tenir compte de la question de ce qu'on appellerait aujourd'hui l'ethnicité des populations concernées et de leur degré très varié d'acculturation par la société chinoise environnante.

Cette étude considère essentiellement le cas des 'Miao' de la province du Guizhou au XVIII ${ }^{\mathrm{e}}$ siècle. Si le contexte général de la transformation administrative mise en œuvre à partir de 1726 sous Yongzheng et des révoltes qu'elle a engendrées est aujourd'hui mieux connu, il semble intéressant d'examiner deux aspects de cette entreprise. Le premier aspect concerne le statut des «Miaoli »-c'est à dire des « lois des Miao », ou des « lois sur les Miao » - au sein de l'architecture d'ensemble des lois éditées par l'État mandchou pour assurer le contrôle de ces populations allogènes, soit une tension entre la nécessaire reconnaissance des pratiques indigènes et la volonté de les réduire progressivement au régime commun de la loi impériale. Le second aspect concerne la lutte contre les trafiquants Han pénétrant dans le Guizhou pour se livrer au trafic d'êtres humains : on y perçoit une tension d'une autre nature, celle qui oppose la volonté de réprimer une criminalité à grande échelle et la nécessité de tolérer, voire de légaliser, certains de ces agissements. La complexité des lois sur l'esclavage et la vente d'êtres humains autant que la prise en compte d'un cycle de rébellions et de répressions militaires imposaient au pouvoir mandchou une approche pragmatique.

Ces questions seront successivement examinées après un bref rappel de leur contexte plus général. 


\section{Les tentatives impériales pour administrer les territoires des Miao : la situation nouvelle des Qing}

Les pratiques examinées ici se situent en grande partie dans ce qu'on appelle le « Territoire des Miao ». Ces Miao ne se réduisent pas aux ethnies ni aux nationalités identifiées sous ce nom à l'époque contemporaine et comprennent des populations fort diverses de la Chine du Sud ${ }^{1}$. Leur territoire est appelé miaojie ou, à partir des Qing, miaojiang (région frontière des Miao). Dans les textes officiels, ces termes s'emploient en un sens large et en un sens plus restreint. Il peut s'agir des cinq provinces où sont concentrées les populations indigènes identifiées sous la catégorie des Miao : le Yunnan, le Guizhou, le Sichuan, le Guangxi et le Huguang ${ }^{2}$. Mais il peut s'agir plus précisément des régions peuplées de "Miao crus » (shengmiao) ou " Miao non assimilés », dans des territoires «non acculturés ou non civilisés » (huawai zhi di), la « Région des Miao de mille $l i$ ( (qianli miaojiang) qui, du Guizhou au Hunan, reste en dehors de l'administration centrale.

L'attitude observée par les Ming et les Qing envers les territoires des Miao diffère de deux manières.

En premier lieu, la vigilance des deux dynasties s'exerce dans des directions opposées. Pour les Ming, le danger principal vient du nord et des peuples de la steppe ${ }^{3}$. En revanche, ils sont militairement moins armés devant les nombreuses révoltes de Miao qui éclatent entre 1385 et $1643^{4}$. Au contraire, l'attention du pouvoir mandchou reste orientée vers le sud, où des partisans légitimistes des Ming ont trouvé refuge auprès de populations peu sinisées et où la résistance perdure pendant plusieurs décennies. De puissants généraux Han ralliés au nouveau régime, comme Wu Sangui, écrasent cette résistance avant de constituer à leur tour une menace et d'être supprimés lors de la rébellion des «Trois feudataires » (sanfan zhi luan) de 1674 à 1681. Parallèlement, dans l'immense territoire des Miao, les révoltes se multiplient mais trouvent désormais devant elles une force militaire implacable.

En second lieu, la politique suivie à l'égard des populations Miao sous ces deux dynasties ne peut être que différente dans ses effets, même si elle obéit à une intention similaire de contrôle par le gouvernement central. Héritiers

1. Pour une possible identification de ces populations, voir Hostetler 2001: 101-114.

2. Pour cette identification, voir Qing Shizong shilu, juan $147\left(12^{\mathrm{e}}\right.$ année de Yongzheng). Cf. Yang $2003: 14$.

3. Les Mongols sont décrits de manière routinière « cherchant toujours à revenir malgré leur recul au Nord ». Cf. Zhang $1974: 2235$ (Mingshi, juan 91, «Bianfang »).

4. Zhang 2012 : 141. 
de l'expérience administrative mongole, les Ming poursuivent le système des « chefs indigènes » (tusi) des Yuan, qu'ils généralisent à d'autres provinces ${ }^{5}$. Mais à la différence de leurs prédécesseurs, les Ming réduisent le pouvoir de ces chefs ainsi que le territoire de leur juridiction en le divisant ou en le rattachant à un autre territoire. Ils commencent à introduire dans certaines régions une structure administrative régulière, composée d'officiers militaires et civils, afin de contrebalancer l'autorité des tusi, dont le mandat héréditaire est subordonné à une nomination officielle par la Cour impériale ${ }^{6}$. De plus, la création de la province du Guizhou en 1413 par l'empereur Yongle a des conséquences considérables pour les Miao et leur territoire ${ }^{7}$. Mais cette politique d'ensemble reste inaboutie.

La radicalité de l'entreprise des Qing est en revanche spectaculaire. Il s'agit d'intégrer les populations allogènes, par la persuasion ou par la force, au sein d'un ordre politique et culturel dominé par une élite mandchoue, fière de ses particularités ethniques et soucieuse d'établir solidement sa suprématie militaire $^{8}$. La politique volontariste lancée par l'empereur Yongzheng entend régler ce problème de manière radicale. Cette politique connue sous le nom de « remplacement des chefs indigènes par des fonctionnaires réguliers » (gaitu guiliu) fait partie d'un ensemble de réformes de grande ampleur attachée à remodeler les pratiques administratives et fiscales ${ }^{9}$. Elle s'étend en réalité sur plus d'un siècle et donne lieu à des pratiques diverses selon les régions concernées. Rappelons que cette politique, lancée dans l'ensemble des territoires «barbares » du Sud-Ouest en 1726, concerne notamment les provinces du Guizhou, du Guangxi, du Yunnan, du Hunan, du Sichuan et du Gansu, où les populations non-Han (et non-mandchoues) sont en majorité. Le contrôle établi sur ces régions sous l'empereur Yongzheng est aussi le produit

5. Les Yuan installent ce système dans les provinces du Shaanxi, du Yunnan, du Huguang et du Sichuan. Cf. Song 1976 : 2305-2326 (Yuanshi, juan 91 « Baiguan »). Les Qing le réorganisent dans sept provinces (le Sichuan, le Yunnan le Guizhou, le Guangdong, le Guangxi, le Huguang et le Shaanxi). Selon le décompte de Zhang Zhongkui, le gouvernement des Ming a nommé 1608 chefs indigènes dans l'ensemble de l'empire. Cf. Zhang $2005: 79$.

6. Le remplacement par les fonctionnaires réguliers des chefs indigènes est applicable dans divers circonstances : au cas où ils sont soupçonnés de tentatives de révoltes, coupables de crimes graves ou n'ont pas de descendant mâle. Cf. Li $2013: 28$.

7. Du $2012: 45-50$.

8. Elliott 2001 .

9. Au sein d'une abondante bibliographie, voir Lombard-Salmon 1972; Giersh 2006: 4363 ; Guy 2013. 
d'une concertation approfondie entre le gouvernement central et de hauts fonctionnaires territoriaux ${ }^{10}$.

Le projet présente de multiples dimensions. Il s'agit de mener à bien la réforme politique et administrative, inaboutie sous les Ming, visant à remplacer l'ensemble des anciens chefs indigènes par des magistrats nommés par le pouvoir central ${ }^{11}$. La réussite de ce projet suppose un encadrement militaire accru, capable d'être entretenu par des ressources locales, et l'instauration progressive d'un système fiscal uniformisé ${ }^{12}$. Le contrôle des passages par les unités militaires spécialisées (les xuntang) doit être minutieusement établi. Le système d'auto-défense et de responsabilité communautaire (baojia) est adapté aux conditions locales, selon la distinction conventionnelle entre les populations restées «crues », et fidèles à leurs coutumes «barbares », et les populations « cuites », au moins partiellement acculturées et soumises désormais à la loi impériale ${ }^{13}$.

Ortai (1680-1745) fut l'un des principaux artisans de la réforme lancée par Yongzheng. Nommé gouverneur-général du Guangxi et du Yunnan en 1725, il définit ainsi sa politique : "On laissera à part les populations Miao "crues" et on fera par ailleurs des groupes de trois foyers, Han ou Yi (barbare), pour en faire les unités responsables de la sécurité au niveau local : ce sont ces unités qui seront sanctionnées en cas de problèmes ${ }^{14} »$. Cette politique comporte aussi une dimension d'ordre culturel (changement des normes vestimentaires et des noms usuels, création d'écoles), dont les résultats resteront inégaux.

10. Wu $2012: 240-60$.

11. Ainsi, selon le tableau dressé par Wang Zhonghan, un certain nombre de préfectures et de sous-préfectures sont installées, sous le règne de cet empereur, dans ces régions précédemment sous-administrées : 23 dans le Yunnan, 19 dans le Guizhou et 13 dans le Guangxi. Voir Wang 2004 : vol. 2, 1389-1392.

12. Zelin 1984.

13. Les récits des missionnaires jésuites français recueillis par le père Du Halde donnent une description de première main de la situation existant sous Kangxi : une partie de la province est compartimentée par des ouvrages de défense et des garnisons visant à contrôler l'accès aux territoires des Miao restés «sauvages », mais ce contrôle reste inefficace et ne peut être financé par les ressources locales. Le Guizhou « est rempli de montagnes inaccessibles, c'est ce qui fait qu'une partie de la province est habitée par des peuples, qui ne se sont jamais soumis à l'empereur, et qui vivent dans une parfaite indépendance des lois de l'empire [...]. Il y a quantité de forts et de places de guerre, où l'on entretient de nombreuses garnisons; mais le tribut qui se tire de la province n'est pas suffisant pour leur subsistance ; ainsi la cour est obligée d'y suppléer, et il n'y a point d'année qu'elle n'y envoie du secours ». $C f$. Du Halde 1735 : tome 1, 255.

14. Mémoire du $20^{\mathrm{e}}$ jour du $3^{\mathrm{e}}$ mois à la $4^{\mathrm{e}}$ année de Yongzheng (1726) dans Zhang 1738 : vol. 9, 49-56, juan 1 : 64b-65a. 
En réalité, cette entreprise va susciter la résistance des populations locales, rendant nécessaires à leur tour de nombreuses expéditions militaires. Ortai doit ainsi avoir recours à l'armée pendant plus de cinq ans et doit lancer une vingtaine d'opérations majeures, se traduisant par la destruction de presque deux mille villages et des massacres collectifs ${ }^{15}$.

Cette entreprise de "pacification » s'accompagne d'efforts pour mieux connaître les coutumes de ces populations, selon une " ethnographie impériale » bien décrite par Laure Hostetler ${ }^{16}$. Cette approche vise à une meilleure articulation entre la manière dont les sociétés Miao résolvent leurs litiges et les obligations que cherchent à imposer le Code impérial.

\section{Les « règles coutumières » des Miao : entre reconnaissance et transformation}

La reconnaissance des coutumes des peuples restés en dehors de la «transformation» selon les règles de la culture dominante (huawai ren), comme les Mongols ou les Tibétains, est une caractéristique de l'État mandchou. Mais cette reconnaissance reste partielle. Elle est même progressivement réduite par la création de lois particulières pour ces peuples et leur territoire, comme les lois sur les Mongols (Menggu lüli), les règlements administratifs régissant le territoire des musulmans (Huijiang zeli) ou des Tibétains (Xining fanyi chengli). Cette pratique s'applique aussi aux Miao mais avec une différence essentielle : contrairement au cas des Mongols, des Tibétains et des musulmans, il n'existe pas de compilation de lois ou de règlements Miao ou sur les Miao.

L'ère de Qianlong (1736-1796) est la grande époque d'une législation établie pour contrôler l'ensemble des territoires habités par des ethnies de culture si différente. C'est le moment où les lois sur les Miao sont massivement promulguées et insérées dans le code des Qing (Daqing lüli). Mais c'est aussi le moment où certains «droits coutumiers des Miao », précédemment reconnus, se voient sensiblement réduits : cette réduction prépare une assimilation progressive de ces populations au peuple ordinaire ( $\mathrm{min}$ ). Ainsi, parmi une vingtaine d'articles additionnels (tiaoli) sur les Miao adjoints au code de 1740,

15. Wang 2004 : 1368-1388. On compte 1398 villages détruits dans les régions de Guangshun, Yong'an et Anshun, 630 dans celles de Guangshun, Dingfan et Zhenning. Voir « Ortai yu 'gaitu guiliu'», http://bbs.tiexue.net/post_2688602_1html. Consulté le 27 mars 2010.

16. Hostetler 2001. 
le terme «Miaoli » ne figure que dans un seul. Cet article mérite d'être cité en raison de son caractère particulier :

Dans le cas où un Miao ou un barbare a commis un crime passible des peines de l'exil militaire, de l'exil simple, de la servitude pénale, de la cangue ou du bâton, le jugement sera conclu dans la province concernée. Il suffit d'envoyer un rapport au ministère des Peines pour vérification. En revanche, cette procédure n'est pas applicable à ceux dont le crime commis est passible de la peine de mort. Il est également interdit de dédommager un tel crime par du bétail et de l'argent. Il faut juger le cas strictement selon la loi et adresser ensuite un mémoire à l'empereur. Tout fonctionnaire qui cache la situation sans en faire rapport à ses supérieurs, ou manipule les circonstances pour pouvoir régler le cas localement, dès que le fait est révélé, sera sanctionné selon la délibération du ministère de la Fonction publique. Toutes les affaires concernant les litiges entre les Miao seront réglées selon les coutumes des Miao (miaoli), il n'est pas nécessaire de les juger selon la loi impériale (guanfa) pour éviter des troubles inutiles ${ }^{17}$.

La compréhension de cette loi exige que soit précisé le statut de ce qui est ici appelé Miaoli.

\section{Le terme Miaoli et sa définition dans le code des Qing}

Le sens de l'expression « Miaoli » peut être approché grâce à trois études récentes d'historiens du droit chinois. Pour Su Qin, « elle désigne essentiellement des droits coutumiers (xiguanfa) des Miao selon lesquels, en cas d'homicide et de blessure, on dédommage la famille de la victime en bétail et en grain ». Cependant, « l'expression concerne seulement une partie de l'ensemble des droits coutumiers des Miao qu'on appelle de manière générale les “mœurs des Miao" (miaosu). Le fait qu'on les désigne comme li montre qu'elles sont devenues une partie des lois impériales à la différence d'autres coutumes ${ }^{18}$. Commentant l'étude de Su Qin, Hu Xingdong précise que « sous les Qing, le terme Miaoli est semblable à celui de miaosu ou de yisu (mœurs des Barbares) : il s'agit toujours des règles coutumières propres à différentes ethnies. La seule différence est que l'appellation de "mœurs" ( $s u)$ est péjorative

17. Zheng et Tian 1999 : 601-602 (juan 37 « Duanzui budang »). Selon Xue Yunsheng, cette loi a été établie en 1725, avant d'être modifiée en 1740. Cf. Xue 1994 : 875.

18. Su 1993. 
alors que celle de "règles" (li) implique une reconnaissance ${ }^{19}$ ». Limitant l'examen à l'Ouest du Hunan, l'étude de Huang Guoxin confronte les normes avec la pratique judiciaire effective et constate que cette reconnaissance n'est que relative : de fait, les règles coutumières des Miao sont utilisées de concert avec les lois impériales au cours d'une longue période ${ }^{20}$.

En tout état de cause, trois aspects paraissent clairs : d'abord, l'expression « Miaoli » désigne bien l'ensemble des règles observées par les Miao en tant qu'elles sont reconnues par l'État impérial, quoique leur contenu ne soit jamais précisément défini ni énuméré ; ensuite, l'espace d'exercice des règles ainsi reconnues est toujours strictement circonscrit ; enfin, le statut juridique de ces normes indigènes ne cesse d'être réduit au cours des XVIII ${ }^{e}$ et XIX ${ }^{\mathrm{e}}$ siècles, avant de disparaître complètement du Code impérial en 1906, lors de la grande réforme juridique de la fin des Qing.

\section{L'évolution du rapport entre « coutumes des Miao » et loi impériale}

On peut saisir le changement d'attitude de l'État impérial à l'égard des règles coutumières Miao en comparant deux périodes : celle de Shunzhi (16441661) et Kangxi (1662-1722), et celle de Yongzheng (1723-1736) et Qianlong (1736-1796).

Les règnes de Shunzhi et de Kangxi semblent tolérer un grand nombre de coutumes pratiquées par les indigènes dans le territoire des Miao. Pour les litiges ordinaires, l'État s'occupe seulement des Miao «cuits » tout en laissant les Miao «crus » pratiquer leurs propres coutumes. En revanche, si les Miao « crus » se rendent coupable de crimes violents, on distingue ceux qui sont commis entre eux et ceux dont sont victimes des populations soumises à l'administration impériale. C'est seulement dans ce dernier cas que l'État intervient.

Ces distinctions sont parfaitement illustrées dans un mémoire de 1665 adressé par Yang Maoxu, le gouverneur du Guizhou. Il suggère deux procédures pour régler les crimes violents commis par les Miao crus : les réprimer par la force militaire quand ils s'en prennent aux populations administrées par l'État, mais les laisser gérer eux-mêmes leurs propres vendettas.

La province du Guizhou se trouve dans une région très montagneuse. Elle est habitée par des populations Miao et $\mathrm{Yi}$, qui ne peuvent se comprendre entre elles

19. Hu 2004.

20. Huang 2011. 
en raison de leurs langues différentes. Elles ne connaissent pas le comportement rituel et ne savent manifester leurs sentiments que par des expressions courroucées, et la vendetta est leur comportement habituel. La méthode pour les gouverner est donc nécessairement différente de celle qu'on utilise en Chine centrale. Il faut les réprimer par l'armée quand elles se rassemblent en bande pour piller et tuer la population administrée par le gouvernement local. Quant aux vendettas pratiquées entre elles, dans leur propre territoire au sein des montagnes et des forêts, sans que cela concerne le territoire sous administration impériale, il suffit de suivre l'ancien usage (jiuli) : on ordonne aux chefs indigènes de jouer le rôle d'intermédiaire pour arranger l'affaire, avant d'envoyer un rapport sur le résultat du jugement, à savoir si les deux parties acceptent de mettre à mort les coupables ou de dédommager les victimes par du bétail et des gens [...]. De manière générale, ces Barbares attachent de l'importance aux biens et méprisent la vie humaine ${ }^{21}$.

La reconnaissance des coutumes relatives à la vendetta des Miao constitue selon ce mémoire un usage impérial déjà ancien : ceci est confirmé par un autre mémoire, rédigé huit ans auparavant par Yang Yongjian, gouverneur de la même province, au sujet d'une vendetta des Miao survenue en 1657.

J'ai examiné l'article additionnel établi par l'empereur (dingli) qui stipule que quand les Miao s'entretuent entre eux, il faut ordonner au chef indigène, qu'il ait été ou non nommé par l'État, d'aller demander en personne à la famille de la victime si elle souhaite être dédommagée par une vie de la famille ennemie, auquel cas on la laisse décapiter un membre de cette famille ; sinon, elle sera dédommagée par une personne vivante appartenant à cette dernière, etc. [...] Selon cet article additionnel concernant la vendetta des Miao, on oblige [le coupable d'homicide] à payer par la remise d'une personne vivante ou de bétail, les frais d'enterrement de la victime dans le cas où le fait de payer par une vie n'est pas exigé [par la famille de cette dernière $]^{22}$.

Ce mémoire confirme l'existence, déjà à cette époque, d'un article additionnel sur la vendetta des Miao. Cet article établit une double distinction entre Miao « cuits » et Miao « crus », d'une part et, d'autre part, entre les violences commises entre Miao ou celles commises à l'encontre de la population administrée par la bureaucratie impériale. Cette distinction est maintenue en

21. Cité par $\mathrm{Hu} 2004$.

22. Ibid. 
1701 par un édit de l'empereur Kangxi ${ }^{23}$, qui est modifié en 1705 pour offrir aux Miao qui le désirent la possibilité de recourir aux tribunaux impériaux lorsqu'ils sont victimes d'agression ${ }^{24}$.

L'époque Yongzheng-Qianlong marque un net changement. Certes, le même principe semble prévaloir sous le règne de Yongzheng ${ }^{25}$, mais il disparaît en 1740, lorsqu'une nouvelle version du code des Qing - le Daqing lüli-entre en vigueur. Il contient l'article qui a été déjà présenté comme le seul où se rencontre le terme " Miaoli ». Désormais, la valeur judiciaire des coutumes des Miao relatives à l'homicide et à la violence n'est plus reconnue. Trois règles sont clairement établies : d'abord, les Miao, qu'ils soient «cuits » ou « crus », ne sont plus autorisés à régler selon leurs propres coutumes les crimes de violence commis entre eux, mais doivent être jugés selon les lois impériales. Ensuite, le Miaoli est donc limité désormais aux litiges ordinaires entre Miao. Enfin, une distinction supplémentaire est faite selon la nature du crime. Si les crimes punissables de la servitude pénale et de l'exil restent l'affaire de l'administration locale, les crimes passibles de la peine capitale doivent être jugés à Pékin.

Dans une telle situation, il est normal de constater une grande variété dans les lois impériales sur les crimes jugés « graves » survenus au sein du territoire des Miao.

\section{Les trois types de crimes concernés}

L'examen du code des Qing de 1740 permet de constater l'existence d'une vingtaine d'articles additionnels (tiaoli) relatives aux $\mathrm{Miao}^{26}$. Elles visent

23. Xi et Shen 1982 [1903], juan 375 (« Shili », section « Huawairen youfan », « Mingli lü»).

24. Si les victimes Miao souhaitent recourir aux autorités locales, ces dernières doivent ordonner aux chefs indigènes d'arrêter les coupables et les punir sévèrement selon les lois impériales.

25. L'édit de Qianglong daté de 1736 stipule notamment que « [puisque] les coutumes Miao sont totalement différentes des peuples de l'intérieur [de l'Empire], désormais, tout procès concernant des litiges entre Miao sera réglé selon les usages coutumiers de ces populations et il ne sera pas requis d'appliquer les lois officielles; en cas de procès impliquant des militaires, des Han et des Miao crus, on laissera les officiers civils traiter de ce qui relève des affaires civiles et les officiers militaires s'occuper de ce qui concerne les affaires militaires. Cf. Hu 2004.

26. Ces articles complémentaires se trouvent principalement dans les parties du code des Qing appelées « ministère des Armées » (Bingbu) et « ministère des Peines » (Xingbu). 
à pénaliser trois grands types de crimes : le pillage en groupe en plein jour (7 articles), l'enlèvement contre rançon (4 articles) et le trafic d'êtres humains (4 articles). Ces articles visent à réprimer des crimes qui sont d'abord le produit des pratiques culturelles des populations Miao, notamment la tradition de la vendetta. On ne dira qu'un mot sur les deux premiers types de crimes, en réservant un développement particulier au troisième : celui-ci, en effet, invite à une réflexion plus générale sur les rapports entre le trafic d'êtres humains et les pratiques de l'esclavage sous les Qing.

La persistance de rassemblements parfois de grande ampleur en vue du pillage en territoire Miao représente un premier souci majeur pour le pouvoir mandchou. Trois articles additionnels ont pour objet de les réprimer ${ }^{27}$. On constate que, si l'objectif premier de ces articles concerne bien le pillage, celui-ci s'accompagne parfois d'homicides et de blessures, et nécessite donc une répression particulière. D'autre part, il est clair que la préoccupation des autorités est avant tout l'échelle de tels rassemblements. Au-delà d'une centaine de pilleurs, la vigilance devient extrême dans des territoires qui restent partiellement insoumis et prompts à la révolte. D'une loi à l'autre, on peut constater un alourdissement de la peine qui passe, par exemple, de la décapitation immédiate à l'exposition de la tête décapitée pour ceux qui sont coupables d'un rassemblement de plus de cent personnes ${ }^{28}$. Malgré la répression, ce type de crime ne cesse de se reproduire au cours des XVIII et $\mathrm{XIX}^{\mathrm{e}}$ siècles. En conséquence, de nouveaux articles complémentaires sont promulgués jusqu'au tout début du XXe siècle.

Outre le pillage, un deuxième type de crime, l'enlèvement contre rançon, est perçu comme caractéristique du comportement des Miao. L'expression « les Miao dressent des embuscades pour capturer quelqu'un » (Miaoren fucao zhuoren) est une abréviation courante de l'article suivant :

Chaque fois que les Miao dressent des embuscades pour capturer quelqu'un dans le but de demander une rançon, en cas de premier délit, le coupable principal sera puni de la décapitation à confirmer par les Assises d'Automne et les complices porteront tous la cangue pendant trois mois avec un tatouage sur le bras. En cas de récidive, les responsables seront tous décapités sans délai et on ne fera pas de distinction entre le coupable principal et ses complices ; ceux qui les suivent porteront deux mois la cangue avant d'être bannis à vie aux frontières. Quant au chef indigène responsable, au cas où il n'était pas informé, il sera sanctionné par le ministère de

27. Zheng et Tian $1999: 388,737,900$.

28. Sur ce phénomène d'aggravation du droit commun par le biais de lois spéciales à caractère régional, voir l'article de Wang Zhiqiang dans ce même numéro. 
la Fonction publique selon le nombre des crimes survenus sous sa juridiction; au cas où il était informé et aurait encouragé le crime, il sera destitué de ses fonctions et puni de 100 coups de bâton. S'il suscite le crime ou collabore pour partager le profit, il sera destitué de ses fonctions et portera trois mois la cangue. Il ne sera pas autorisé à racheter sa peine ${ }^{29}$.

Ce type de crime conserve malgré tout une dimension locale, au sein du territoire des Miao. Il est avant tout l'expression de la pratique de la vendetta qui oppose les indigènes entre eux. Il n'en va pas de même de la troisième catégorie de crime, le trafic d'êtres humains organisé. En effet, cette activité criminelle est par définition supra-régionale : elle dépasse les frontières des territoires Miao et peut affecter l'ordre public dans plusieurs provinces à la fois. De plus, c'est un comportement criminel qui soulève la question de l'existence légale de l'esclavage et celle de la vente autorisée d'êtres humains sous les Qing. Il mérite, à ce titre un examen plus attentif.

\section{Le combat contre le trafic d'êtres humains aux frontières des Miao : entre criminalisation et légalisation}

Le trafic d'êtres humains aux frontières du territoire des Miao est à l'origine de combats particulièrement coûteux menés par le gouvernement mandchou lorsqu'il décida d'imposer l'administration directe à ces populations allogènes au cours du XVIII ${ }^{e}$ siècle. Ce trafic existait longtemps avant la réforme de Yongzheng. Mais il était considéré par beaucoup d'artisans de cette réforme comme un des obstacles majeurs à sa réussite dans le territoire des Miao.

\section{La découverte d'un trafic multi-ethnique}

Dès le début de la réforme, en 1724, Gao Qizhuo, alors gouverneur général du Yunnan et du Guizhou, consacre une analyse à ce problème, dans un de ses premiers rapports adressés à l'empereur:

29. Cet article complémentaire est déjà intégré dans le code de 1725. Zheng et Tian 1999 : 403. 
Parmi les Miao du Guizhou, les Zhong ${ }^{30}$ sont particulièrement brutaux et mauvais, mais d'autres Miao commettent également de manière fréquente le pillage. On a dit que c'est dans la nature des Miao de s'adonner à la vendetta et au goût de tuer. J'ai vérifié ce phénomène de près. En fait, ce comportement est très lié à la recherche du profit. Ce même motif est à l'origine de la vendetta et du goût pour la tuerie. Ils tuent pour prendre les biens de leur victime. Dès qu'ils ont vu chez autrui des objets en argent, l'idée de les piller leur vient immédiatement à l'esprit, elle ne les abandonne pas avant que ces objets n'aient été obtenus.

Cependant, les gens du Guizhou sont généralement pauvres et peu d'entre eux possèdent des objets en argent qu'on puisse leur dérober. De là le projet d'enlever des êtres humains [pour se procurer une rançon] qui éveille chez les Miao crus le goût du pillage et du meurtre. [Car] la province du Guizhou partage des frontières avec celle du Sichuan où le prix de vente d'un être humain est considérablement plus élevé. Les trafiquants venus du Sichuan demandent souvent aux malfaiteurs locaux du Guizhou de collaborer avec des Miao pour enlever des personnes en vue d'en tirer profit en les vendant. Les Miao obtiennent quatre à cinq taels d'argent pour vendre une personne à un trafiquant local ; ce dernier obtient plus de dix taels en la revendant à un trafiquant du Sichuan et celui-ci, à son tour, obtient plus de vingt taels en la revendant dans le Sichuan. Aussi tous les Miao se disputent-ils pour s'engager par tous les moyens dans cette entreprise aussi profitable.

À mon avis, la moitié des pillages et des homicides commis par ces Miao sont dus à la vendetta traditionnelle et l'autre moitié sont commis pour de l'argent. Si l'on arrivait à faire cesser ce trafic d'êtres humains, les pillages et les meurtres commis par les Miao seraient réduits de moitié $[\ldots]^{31}$.

Peu convaincu par les explications culturelles sur la vendetta, ce gouverneur souligne la motivation économique de ces comportements, essentiellement dus à une conjoncture nouvelle, qui offre des occasions de profit. Il existe une grande disparité entre la pauvreté des ressources du territoire Miao et le potentiel d'un marché voisin, celui du Sichuan. En effet, le Sichuan a connu un dépeuplement spectaculaire ${ }^{32}$. En conséquence, la demande en hommes est

30. Les Zhongjia sont aujourd'hui identifiés à la "minorité nationale" Buyi qui parle une langue du groupe linguistique Tai. Sur la situation de leur territoire sous les Qing, en particulier lors de la révolte de 1797, voir Weinstein 2013.

31. Yongzheng chao hanwen zhupi zouze huibian 1990 : vol. 3. 82-83.

32. Selon une estimation, vers 1680 la province avait perdu des deux-tiers aux trois-quarts de sa population après une quarantaine d'années de combats continus. $C f$. Dai 2010 : 27. Cette situation a rendu nécessaire un vaste projet de migration, particulièrement important sous Kangxi. 
forte dans cette province : elle suscite une offre chez son voisin, car le prix d'un être humain est beaucoup plus élevé au Sichuan qu'en territoire Miao. La présence croissante de groupes de Han dans le Guizhou rend possible une collaboration transrégionale.

Ce qui inquiète les dirigeants, c'est aussi bien l'importance des complicités locales devant ces perspectives lucratives (chefs indigènes, soldats, commis du gouvernement local) que l'ampleur du trafic ainsi généré à une échelle interprovinciale. Un passage du même rapport précise encore l'organisation de cette criminalité désormais multiethnique :

Les Miao et les trafiquants du Sichuan se mettent d'accord au préalable sur les prix fixés pour les garçons de plus de dix ans et ceux de moins de dix ans, ainsi que sur les prix fixés pour les femmes de différentes tranches d'âge. Ensuite les Miao rassemblent une bande et se cachent dans le sous-bois. Quand ils voient des voyageurs, de jeunes bergers ou des femmes qui ramassent du petit bois, ils s'en emparent et les emmènent chez eux. Ils les négocient ensuite un à un avec les trafiquants du Sichuan. Après quoi ceux-ci, accompagnés par les Miao, sortent de la province en se cachant le jour et voyageant la nuit à travers forêts et montagnes ${ }^{33}$.

Les trafiquants Han sont reconnus comme les principaux responsables de ces réseaux de criminalité lors des campagnes militaires d'Ortai, gouverneur du Yunnan et du Guizhou, à partir de 1726. Ils sont régulièrement capturés et condamnés à la décapitation immédiate, ou à la strangulation à confirmer par les Assises d'automne ${ }^{34}$.

En dépit de cette répression, le trafic continue à prospérer. En 1738, dix ans après les opérations menées par Ortai, Chen Derong, commissaire du Guizhou, souligne que ce trafic de nature commerciale devient particulièrement préoccupant lorsqu'il se produit dans un contexte politique de révoltes : « $\mathrm{Ce}$ trafic est rendu encore plus intense du fait des révoltes des Miao, et on rencontre toutes sortes de cas aussi étranges qu'effrayants : certains tuent le mari pour enlever l'épouse, d'autres massacrent les parents pour enlever des enfants ${ }^{35}$. »

\section{Ibid.}

34. Voir en particulier le rapport d'Ortai, daté de 1726, sur les complicités existant entre Han, Miao et Miaoluo (les Yi d'aujourd'hui) : « Yungui zongdu Ertai wei chengji souhuo chuanfan shi zouzhe » (Mémoire d'Ortai, gouverneur-général du Yunnan et du Guizhou, sur la capture des tranfiquants provenant du Sichuan en profitant de l'occasion [de la représsion à Changzhai]), dans Ha 2009 : 3-15.

35. Ren, Yang et al. 1988 : vol. 3, 315. 
Il reste que, pour comprendre la difficulté et la relative inefficacité du combat contre ces comportements criminels, il faut prendre en compte un autre facteur : la contradiction entre la mise hors la loi de ce trafic et la tolérance relative envers l'esclavage et la vente d'êtres humains sous les Qing.

\section{Les ambiguïtés de la pratique : légalisation $v s$ criminalisation}

La situation de l'esclavage sous les Qing est particulière en raison des différences culturelles entre les traditions mandchoues et les pratiques suivies en Chine jusqu'aux Ming. L'État mandchou a d'abord été un " khanat » suivant le modèle de l'Asie centrale, où la majorité des esclaves était la propriété personnelle du souverain, avant de s'adapter progressivement à la situation des populations conquises ${ }^{36}$. Elle est aussi rendue complexe par la difficulté d'appliquer dans ce contexte les catégories européennes concernant les populations « libres » et «non libres » : on rencontre de multiples degrés de servitude.Enfin, il existe des disparités entre ce que la loi reconnaît officiellement et la réalité des pratiques effectives : ainsi, d'une manière générale, le code des Qing ne reconnaît pas le droit au peuple ordinaire (shumin) de posséder des esclaves (nubi), mais la multiplication des articles additionnels à ce sujet laisse percevoir la variété des situations concrètes.

En principe, une personne considérée comme libre ne peut être asservie, et ce statut servile ne concerne que quatre catégories de sujets : les populations capturées, les condamnés, les descendants d'esclaves et les personnes qui se vendent volontairement ${ }^{37}$. Dès lors que la loi autorise quelqu'un à se vendre, ou à vendre des membres de sa famille, en cas de situation extrêmement misérable (pauvreté, famine, etc.), il existe un marché d'êtres humains limité mais légal. Il faut prendre en compte le fait général que la possession de personnes est négociée selon deux mécanismes différents : celui de la loi de l'État et celui des contrats entre particuliers. Le mandarin local est chargé de veiller à la régularité de ces derniers, qui relèvent des ces « affaires mineures » sur lesquelles il a un pouvoir discrétionnaire, seule les « affaires graves » étant transmises aux instances supérieures pour révision et vérification ${ }^{38}$. Toutefois, l'État mandchou impose indirectement son contrôle sur ce marché d'êtres humains en rendant obligatoire la vente d'une personne selon un contrat respectant des règles formelles : il doit être volontairement signé par les deux

36. Pour un tableau général, voir Crossley 2011 : vol. 3, 186-214.

37. Kang Yong Qian shiqi chengxiang renmin fankang douzheng ciliao 1979 : 359-389.

38. Crossley 2011. 
parties et un intermédiaire professionnel (yaren), avant d'être officiellement estampillé par les autorités. On observe que cette réglementation devient plus systématique à partir des règnes de Yongzheng et de Qianlong. Voici un contrat de ce type rédigé en 1724 :

Le contractant de la vente soussigné Gao San, originaire du village des Zhu dans le district Xianghe, établit aujourd'hui ce contrat écrit. En raison d'une pauvreté extrême et de la difficulté de subvenir à mes besoins, je confie volontairement à un intermédiaire le soin de vendre à Wang Chunzhai comme domestiques, moimême, Gao San, âgé de 35 ans, mon épouse née Wu, âgée de 29 ans, Quan'er, mon fils aîné, âgé de 12 ans et mon fils cadet, âgé de 7 ans, soit quatre personnes au total. Le prix négocié pour cette vente est de 15 taels d'argent et il a bien été reçu le jour de la vente. Désormais nous reconnaissons que notre maitre pourra nous renommer et nous donner ses ordres, sans qu'il soit possible d'y trouver à redire à cause d'un statut légalement non éclairci. S'il arrive un événement imprévu, nous l'accepterons comme une marque du destin [sans remettre en question ce contrat]. En cas de fuite ou de disparition, mon beau-père, mes frères aînés et le garant en assureront la responsabilité. Les deux parties sont volontaires et ne reviendront pas sur leur parole. En témoignage de quoi, nous établissons ce contrat de vente complet pour qu'il puisse être consulté. Le $11^{\mathrm{e}}$ mois de la

$2^{\mathrm{e}}$ année de Yongzheng Wu Liangmei, le beau-père Gao Da et Gao Er, frères aînés Gao San, contractant de la vente Erxiao, cousin Liu Guorong, le garant ${ }^{39}$.

Dans les faits, l'existence de ce marché semi-légal permet l'acquisition d'une personne par contrat privé sans toujours la déclarer. Cette pratique perdurera jusqu'en 1910, date de l'abolition officielle du statut de servitude.

C'est cet espace juridique laissé ouvert, entre la stricte prohibition de l'esclavage et la tolérance envers une forme de servitude volontaire, qui explique la difficulté du combat des gouverneurs contre le trafic d'êtres humains au Guizhou. En raison de la réforme engagée par Yongzheng, les fonctionnaires de l'empire sont confrontés localement à un trafic à grande échelle et de rébellions répétées. Fort pragmatiquement, l'État renforce les contrôles sur les trafiquants Han et assouplit les conditions de vente lorsqu'il s'agit des populations Miao. La proposition faite par Gao Qizhuo, en 1724,

39. Wei $1982: 42$. 
revient à légaliser partiellement une situation de fait : «Pour les gens venant d'autres provinces qui souhaitent acheter des garçons ou des filles de familles pauvres [au Guizhou], le nombre des personnes achetées ne doit pas dépasser quatre ou cinq et le contrat de vente doit être scellé par les autorités locales ; ceux qui dépassent cette limite seront punis selon l'article additionnel sur le rapt et la vente des personnes ${ }^{40}$. »

Cette proposition est acceptée par l'empereur Yongzheng. Elle sera finalement ajoutée au code de 1740 en ces termes :

Tous les gens du bon peuple venus d'autres provinces qui achètent des garçons et filles de familles pauvres au Guizhou doivent en faire déclaration aux autorités locales qui appliquent leur sceau sur le contrat de vente. Toutefois, nul n'est autorisé à acheter plus de quatre à cinq personnes. Il faut établir un contrat officiel pour ces dernières auprès des autorités de la préfecture et de la sous-préfecture dans la province d'accueil. Pour tous ces hommes et femmes mis en vente, il faut qu'un garant reconnu par les autorités locales les interroge sur leur origine, fixe un prix, et établisse un contrat sur lequel sont indiqués leur patronyme et leur nom personnel, le lieu de leur domicile, leur sexe et leur date de naissance, avant de le faire estampiller. Toute personne impliquée dans un tel commerce doit être enregistrée dans le cahier fiscal qui circule mensuellement pour percevoir les impôts (xunhuan yinbu). Si le contrat de vente ne porte pas le sceau officiel ou si le nombre des personnes achetées est supérieur à trois, les gens impliqués dans cette affaire seront poursuivis en vertu de l'article sur le rapt ou la vente de personnes. Le même article sera appliqué au garant officiel s'il collabore avec des trafiquants ou s'il ne fait pas apposer le sceau officiel sur le contrat de vente par les autorités. Si le garant officiel soutire de l'argent aux acheteurs pour une vente légale, ces derniers sont autorisés à le dénoncer auprès du yamen afin qu'il soit puni. Si les mandarins locaux ne vérifient pas en toute clarté l'origine des personnes concernées, qu'il s'agisse des Miao ou du bon peuple, d'hommes ou de femmes, et apposent leur sceau sur le contrat pour autoriser la vente aux trafiquants venus du Sichuan, ils seront sanctionnés également selon ce même article additionnel. Quant aux Miao possesseurs d'un contrat de vente dûment scellé, il faut leur donner un passeport sur lequel sont inscrits leur nom, âge et caractéristiques physiques pour que les chargés de contrôle militaire ou civil puissent les laisser passer. Si ces derniers les retiennent pour leur soutirer de l'argent ou reçoivent des pots de vin pour laisser passer des trafiquants illégaux, ils seront poursuivis selon la loi et leurs supérieurs, civils ou militaires, seront sanctionnés ${ }^{41}$.

40. Ren, Yang et al. $1988:$ vol. 3, 177.

41. Zheng et Tian 1999: 407. 
D'un autre côté, au moment même où il autorise la vente de personnes à des acheteurs ordinaires dans le Guizhou, l'État impérial poursuit son combat contre les réseaux de trafiquants pour imposer son contrôle. Il confirme ainsi en 1725 un ancien article additionnel, qui existait déjà sous Kangxi :

Dans la région du Guizhou, des malfaiteurs provenant d'autres provinces, agissant en connivence avec des hors-la-loi locaux, enlèvent des hommes et des femmes du peuple, et vont jusqu'à s'emparer des habitants de villages désertés et reculés pour les vendre au Sichuan et au Huguang. Le coupable principal sera condamné à la décapitation immédiate, selon l'article complémentaire sur le pillage ou l'enlèvement, fait en bande, de femmes en déplacement, et exécuté à l'endroit où le crime a été commis. Les complices seront condamnés à la strangulation à confirmer par les assises d'automne ${ }^{42}$.

Cet article additionnel est complété par un autre, visant plus spécialement les collaborateurs et recéleurs des trafiquants :

Pour ceux qui donnent asile aux trafiquants du Sichuan, au cas où il est établi et avéré qu'ils ont guidé ces trafiquants, enlevé des gens et les ont recelés avant de les vendre, ils seront condamnés à la décapitation sans délai, selon la loi sur le coupable principal pratiquant un commerce illégal. Avec le coupable principal parmi les trafiquants du Sichuan, ils doivent être décapités sur le lieu du crime $[\ldots]^{43}$.

La relative tolérance envers la vente de personnes et la criminalisation du trafic privé au Guizhou sont deux faces d'un même combat impérial : imposer le pouvoir judiciaire sur ce territoire afin de rester maître du trafic d'êtres humains qui s'y donne cours. Cette attitude est aussi encouragée par la recherche d'expédients, dus aux effets de la situation militaire.

Les forces militaires des Qing ont été mobilisées pendant sept ans pour reconquérir les territoires Miao, après les révoltes suscitées par la réforme de Yongzheng. Ces opérations ont eu pour effet la capture d'une vaste population, réduite ensuite en esclavage. Ainsi, à l'occasion d'une seule bataille menée en 1736 par Zhang Guansi à Niupi dajing, plus de 13600 Miao sont faits prisonniers et sont donnés aux officiers et aux soldats comme esclaves ${ }^{44}$. L'argumentaire de ce gouverneur est pragmatique : il est peu praticable, selon lui, de suivre les règlementations qui demanderaient qu'on escorte les

42. Wu 1992 : 754 (« Lueren luemairen yishan liwen »).

43. Zheng et Tian $1999:$ 407-408.

44. Qingdai qianqi miaomin qiyi dang'an shiliao huibian $1987: 210$. 
prisonniers jusqu'à une autre province pour les y installer car la route, à la fois longue et difficile, conduirait à des pertes de vies humaines. D'autre part, les autorités locales ne sont pas capables d'assurer la logistique, et notamment le ravitaillement nécessaire. De plus, si on regroupe dans les garnisons une telle population qui comprend beaucoup de femmes, cela nuira au moral des forces armées. En conséquence, on propose de « les vendre pour récompenser des soldats de mérite ${ }^{45}$ ». Dès lors, les autorités tant civiles que militaires ne peuvent que participer à ce vaste trafic de Miao.

On reconnaît le danger d'une telle situation : «Les trafiquants du Sichuan et du Guizhou viennent en groupe aux garnisons » et « les transforment en marché ou lieu de trafic ${ }^{46}$. De plus, on a été conduit à fixer un tableau de prix officiel organisant ce trafic, comme l'indique le gouverneur du Hunan, Xu Rong, qui propose de l'adopter dans sa province: « un taël d'argent pour une fille Miao de trois à cinq ans ; deux taëls pour celle de six à onze ans ; trois taëls pour celle de 12 à 16 ans ; cinq pour celle de 17 à 30 ans ; deux taëls pour une femme de 30 à 40 ans ; cinq sapèques pour celle de plus de 40 ans, des personnes âgés et faibles ainsi qu'un bébé d'un an à deux ans ${ }^{47}$. »

La participation du gouvernement et de l'armée impériale au trafic des Miao capturés rend problématique la volonté de contrôler ce commerce selon la loi. Elle encourage au contraire son extension. Ainsi, vingt-six ans après la promulgation sous Yongzheng d'articles réprimant spécialement ce fléau au Guizhou, un nouvel article de 1751 étend la répression au Yunnan et au Sichuan. Les peines sont alourdies à l'encontre de crimes présentés comme encore plus violents. Voici cet article :

Dans la région du Guizhou, les individus venus d'autres provinces, qui agissent en connivence avec des malfaiteurs locaux pour tuer des habitants Han ou Miao de villages désertés et reculés afin d'enlever leurs femmes et leurs enfants dans le but de les vendre, seront tous punis, selon la loi principale sur le vol avec violence, de la décapitation avec la tête exposée. On ne fera aucune distinction entre les coupables principaux et secondaires, et on ne prendra pas en considération le fait que la vente ait eu lieu ou non, pas plus que le fait que les personnes enlevées aient ou non déjà franchi les frontières $[. .$.$] .$

Quant à ceux qui enlèvent de force des gens ou usent de stratagèmes pour s'en emparer par séduction afin de les vendre au Sichuan, le coupable principal sera décapité sans délai et les complices seront décapités après les assises d'automne.

45. Ibid.

46. Ibid. : 182 .

47. Ibid. : 430 . 
On ne prendra pas en considération le fait que la vente ait eu lieu ou non, pas plus que le fait que les personnes enlevées aient ou non déjà franchi les frontières [...]. Pour ceux qui accordent l'asile aux trafiquants du Sichuan, s'ils ont guidé ces derniers à tuer pour enlever des gens ou coopéré à leurs stratagèmes pour s'emparer d'enfants par voie de séduction et les garder afin d'en faire le trafic, et si tout cela est bien établi et avéré, ils seront condamnés à la décapitation sans délai comme les principaux coupables [...]. Les autorités locales du Yunnan et du Sichuan appliqueront cette même loi en cas de crimes relatifs au rapt et trafic de personnes. On donne un délai d'une année pour arrêter les trafiquants. Si les préposés militaires ou civils chargés de l'arrestation dépassent ce délai, ils seront sanctionnés en tenant compte du nombre des trafiquants concernés ${ }^{48}$.

On perçoit les trois domaines dans lesquels l'État souhaiterait contrôler une situation qui semble toujours lui échapper. Il s'agit d'abord d'une question d'échelle : il n'est pas concevable qu'un nombre exagéré de personnes de condition servile soit possédé par des particuliers, d'autant plus que cette condition les soustrairait à l'administration fiscale. Il s'agit aussi du statut d'origine des populations concernées : la vérification par les fonctionnaires locaux doit établir s'il s'agit de Miao ou de Han, de gens du bas peuple ou au contraire du bon peuple, etc. Enfin, sans que ce soit toujours très clair, l'attention portée au contrat implique aussi que cette vente garde une apparence volontaire. Certes, dans le cas de prisonniers Miao réduits en esclavage par l'État, leur mise en vente est une peine qui ne suppose pas le consentement. Mais dès lors que des particuliers sont ainsi encouragés à les acheter pour les transférer dans d'autres provinces, la situation est probablement plus floue aux yeux des autorités dans les régions d'accueil. Comment de telles transactions se passaient-elles, concrètement ? Il faudrait des recherches supplémentaires pour y répondre. Dans une situation ordinaire, la vente légale suppose un consentement des parties, enregistré dans un contrat. Sinon, il s'agit d'un crime (le rapt par la violence ou la séduction) qui est puni par la loi. Mais il existe de toute évidence une vaste « zone grise » où des vendeurs et acheteurs d'esclaves peuvent procéder à leurs transactions sans respecter la lettre de la loi, ou en donnant à leurs agissements une apparence légale.

48. Voir la version chinoise électronique http://lsc.chineselegalculture.org/eC/ HDSLXB/2.6.17.286.0 


\section{Conclusion}

La conquête des territoires des Miao au XVIII ${ }^{\mathrm{e}}$ siècle s'est accompagnée d'une reconnaissance limitée de certaines « règles coutumières » des Miao, mais aussi par l'imposition de plus en plus rigoureuse des lois impériales au sujet des Miao. Pour imposer son monopole sur la violence au sein de ces nouveaux territoires, l'État impérial a organisé des campagnes militaires ou policières successives au cours de plus d'un siècle. Ces campagnes sont dirigées contre trois grands crimes attribués aux Miao «crus »: le pillage, l'enlèvement contre rançon et le trafic d'êtres humains.

Si les deux premiers crimes sont limités aux territoires des Miao et concernent le plus souvent ces populations elles-mêmes, le troisième crime dépasse cette dimension ethnique et territoriale et affecte l'ordre et l'intérêt impérial dans plusieurs provinces. Au cours des combats contre ce dernier crime, le législateur mandchou fait face à un double dilemme : maintenir le privilège originellement propre aux gens des Bannières de posséder des esclaves tout en permettant la possession limitée de gens de conditions servile par de simples particuliers chinois ; ou bien criminaliser le trafic d'êtres humains organisé par les trafiquants, alors même qu'ils passent par eux pour vendre les populations Miao capturées par l'armée impériale. Le résultat en est une politique pragmatique autorisant dans les faits ces trafiquants illégaux à avoir accès à ce marché de Miao réduits en esclavage.

Dès lors, il n'est peut-être pas exagéré de dire que l'imposition de l'administration directe par le gouvernement central signifie aussi un processus d'asservissement d'une partie de la population Miao ainsi qu'un aval donné à des particuliers pour la vente d'êtres humains. C'est cette contradiction qui a rendu le combat contre le trafic d'êtres humains si difficile. Après l'époque de Qianglong, les articles sur ce sujet se multiplient, leur énoncé évoque moins l'ethnicité et davantage le caractère régional ou provincial, mais leur nombre même indique la permanence des comportements criminels autour de ce commerce lucratif. 


\section{BIBLIOGRAPHIE}

\section{Sources}

Qingdai qianqi miaomin qiyi dang'an shiliao huibian (Compilation des archives sur les révoltes des Miao au début des Qing) (1987). Compilé par les Archives historiques $\mathrm{n}^{\mathrm{o}} 1$ de la Chine, Pékin : Guangming ribao chubanshe.

Kang Yong Qian shiqi chengxiang renmin fankang douzheng ziliao (Documentation des révoltes des populations en ville et dans la campagne sous le règne de Kangxi, Yongzheng et Qianlong) (1979). Compilé par l'Institut d'études des Qing à l'Université du peuple, Pékin: Zhonghua shuju.

Yongzheng chao hanwen zhupi zouze huibian (Recueil des mémoires rédigés en chinois avec les réponses officielles en caractères rouges de l'empereur Yongzheng) (1990). Nanjing : Jiangsu guji chubanshe.

SoNG, Lian (1976). Yuanshi (Histoire des Yuan). Pékin : Zhonghua shuju.

Wu, Tan (1992). Daqing lüli tongkao. In Daqing lüli tongkao jiaozhu, édité par MA Jianshi et YANG Yushang. Pékin : Zhongguo Zhengfa daxue chubanshe.

XI, Yufu et SHEN, Shixu, éd. (1982 [1903]). Huangchao zhengdian zuan. Réimpression : Taipei : Wenhai.

XuE, Yunsheng (1994). Duli cunyi (Doutes persistants à la lecture des articles complémentaires du Code). Ponctué et édité par Hu Xingqiao et DENG Youtian. Pékin : Zhongguo renmin gong'an daxue.

ZHANG, Tingyu et al. (1974). Mingshi (Histoire des Ming). Pékin : Zhonghua shuju.

ZHANG, Tingyu (1738 [1732]). Zhupi yuzhi (Édits impériaux en caractères rouges). Édition de Qing neifu keben. vol.9, juan 1.

ZHENG, Qin et TIAN Tao (1999). Daqing lüli (Code des Grands Qing). Beijing : Falü chubanshe.

\section{Études}

Crossley, Pamela Kyle (2011). «Slavery in Early Modern China. » In The Cambridge World History of Slavery, vol. 3. Cambridge, Cambridge University Press : 186214.

DAI, Yingcong (2010). The Sichuan Frontier and Tibet : Imperial Strategy in the Early Qing. Seattle : University of Washington Press.

DU, Huailiang (2012). «Shi Shu 'gaitu guiliu yu Guizhou jiansheng de guanxi » (À propos du rapport entre la politique visant à « remplacer les chefs indigènes par des fonctionnaires réguliers » et la création de la province du Guizhou). Guizhou shehui zhuyi хиеуuan xuebao, 3: 45-50.

Du Halde, J.-B. (1735). Description géographique, historique, chronologique, politique et physique de l'Empire de la Chine et de la Tartarie chinoise. Paris: J.-B. Mercier.

ELlioTt, Mark C. (2001). The Manchu Way: The Eight Banners and Ethnic Identity in Late Imperial China. Stanford : Stanford University Press. 
GIERSH, C. Patterson (2006). Asian Borderlands, The Transformation of Qing China's Yunnan Frontier. Cambridge : Harvard University Press.

GuY, R. Kent (2013). Qing Governors and their Provinces : The Evolution of Territorial Administration in China, 1644-1796. Seattle : University of Washington Press.

HA, Enzhong (éd.) (2009). « Yongzheng nianjian zhengchi Guizhou chuanfan shiliao » (Documents sur les opérations contre des trafiquants provenant du Sichuan au Guizhou pendant le règne de Yongzheng). Lishi dang'an, 4 : 3-15.

HosteTLER, Laure (2001). Qing Colonial Entreprise : Ethnography and Cartography in Early Modern China. Chicago/Londres : The University of Chicago Press.

HU, Xingdong (2004). « Qingdai minzufa zhong 'miaoli' zhi kaoshi » (Examen et interprétation des Miaoli dans les lois des Qing sur des ethnies). Sixiang zhanxian, $6: 33-38$.

HUANG, Guoxin (2011). «'Miaoli’ : qing wanhchao hunan xinkai miaojiang diqu de falü zhidu anpai yu yunzuo shijian (Le Miaoli, une structure institutionnelle juridique et la pratique de l'empire des Qing dans le territoire nouvellement conquis au Hunan). Qingshi yanjiu, $3: 37-47$.

LI, Wengang (2013). «Qingdai tian dongbei gaituguiliu yanjiu zhi wenxian huigu yu zhanwan » (Rétrospectives et perspectives des recherches sur la politique visant à remplacer les chefs indigènes par des fonctionnaires réguliers lancée par le gouvernement des Qing dans le Nord-Est du Yunnan). Journal of Zhaotong University, 35.1: 28-32.

LOMBARD-SALMON, Claudine (1972). Un exemple d'acculturation chinoise. La province du Guizhou au xviie siècle. Paris : Publications de l'École Française d'Extrême-Orient.

REN, Kecheng, YANG Enyuan et al. (1988). Guizhou tongzhi (Monographie générale $d u$ Guizhou). Guiyang : Guizhou renmin chubanshe.

SU, Qin (1993). « Miaoli kaoxi » (Un examen analytique de Miaoli). Minzu yanjiu 6 : 97-103.

WANG, Zhonghan (2004). Wang Zhonghan qingshi lunji (Collections de travaux de Wang Zhonghan sur l'histoire des Qing). Pékin : Zhonghua shuju.

WEI, Qingyuan (1982). Qingdai nubi zhidu (Le système d'esclavage sous les Qing). Pékin : Renmin daxue chubanshe.

WeInStEIn, Jodi (2013). Empire and Identity in Guizhou : Local Resistance to Qing Expansion. Seattle: University of Washington Press.

WU, Guo (2012) « Accommodation and Conflict : The Incorporation of Miao Territory and Construction of Cultural Difference of Miao Territory during the High Qing Era. » Frontiers of History in China, 7: 240-260.

YANG, Shengyong (2003). Qingchao jingying guizhou miaoqiang yanjiu (Étude sur l'administration du territoire des Miao au Guizhou sous les Qing). Thèse de doctorat, Pékin : Université Centrale des nationalités.

ZELIN, Madeleine (1984). The Magistrate's Tael: Rationalizing Fiscal Reform in Eighteenth Century Ch'ing China. Berkeley : University of California Press.

ZHANG, Xiaosong (2005). «Lun Yuanmingqing shiqi de xinan shaoshu minzu tusi, tuguan zhidu yu gaitu guiliu » (Étude sur le système des chefs indigènes dans le 
Sud-Ouest sous les dynasties Yuan, Ming et Qing). Zhongguo bianjiang shidi, $15.2: 78-84$.

ZHANG, Zhongkui (2012). Gaitu guiliu yu miaojiang zaizao-Qingdai 'xinjiang liuting' de wanghua jincheng jiqi shehui wenhua bianqian (La politique visant à « remplacer les chefs indigènes par des fonctionnaires réguliers » et le remodelage du territoire des Miao : Le processus d'assimilation de six préfectures créées dans le territoire nouvellement conquise et leur transformation socioculturelle). Pékin : Zhongguo shehui kexue chubanshe.

\section{GlosSAIRE}

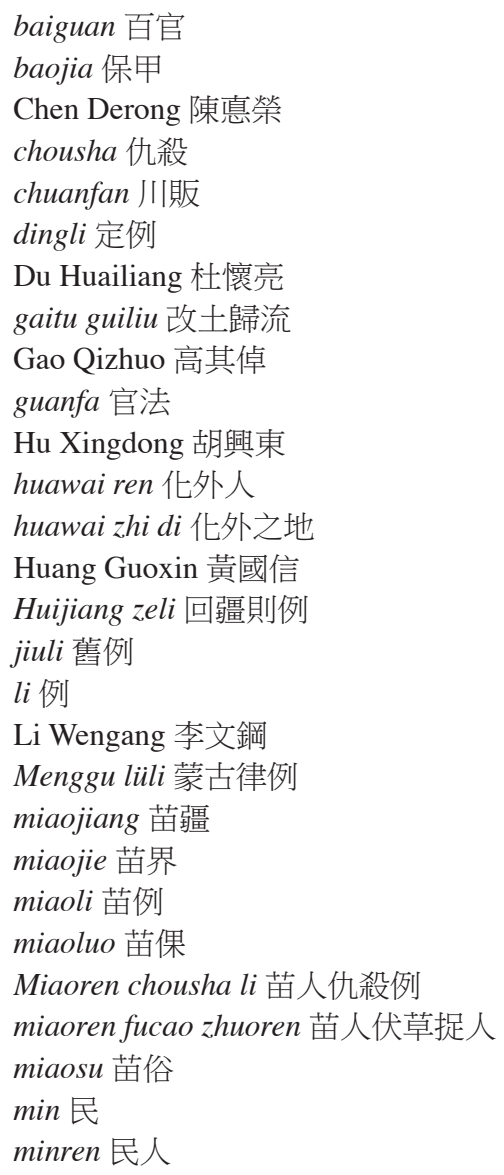


Zhang Ning (Laure)

Niupi dajing 牛皮大箐

nubi 奴婢

Ortai 鄂爾泰

qianli Miaojiang 千里苗疆

sanfan zhi luan 三藩之亂

shumin 庶民

$s u$ 俗

Su Qin 蘇欽

tusi 土司

Wu Sangui 吳三桂

xiguanfa 習慣法

Xining fanyi chengli 西寧番夷成例

xunhuan yinbu 循环印簿

xuntang 汗塘

yaren 牙人

Yang Maoxun 楊茂勛

Yang Shengyong 楊勝勇

Yang Yongjian 杨雍建

Xu Rong 許容

yi 彝

$y i s u$ 夷俗

Zhang Guangsi 張廣泗

Zhang Xiaosong 張曉松

Zhang Zhongkui 张中奎

zhong 仲 